\title{
Curcumin enhances the radiosensitivity in nasopharyngeal carcinoma cells involving the reversal of differentially expressed long non-coding RNAs
}

\author{
QIRUI WANG $^{1^{*}}$, HAONING FAN $^{1 *}$, YING LIU $^{2 *}$, ZHIXIN YIN $^{3}$, HONGBING CAI $^{1}$, JIE LIU $^{2}$, ZHIYUAN WANG $^{2}$, \\ MENG SHAO $^{1}$, XUEGANG SUN ${ }^{1}$, JIANXIN DIAO ${ }^{1}$, YUANLIANG LIU ${ }^{1}$, LI TONG ${ }^{1}$ and QIN FAN ${ }^{1}$ \\ ${ }^{1}$ College of Traditional Chinese Medicine, ${ }^{2}$ Department of Radiotherapy, NanFang Hospital, \\ ${ }^{3}$ Institute of Genetic Engineering, Southern Medical University, Guangzhou 510515, P.R. China
}

Received October 22, 2013; Accepted December 2, 2013

DOI: $10.3892 /$ ijo.2013.2237

\begin{abstract}
Long non-coding RNAs (lncRNAs) are aberrantly expressed and have important functions in pathological processes. The present study investigated the lncRNA profiles and the effects of curcumin (Cur) on the radiosensitivity of nasopharyngeal carcinoma (NPC) cells. The lncRNA and mRNA profiles of each cell group were described by microarray analysis. Numerous differentially expressed genes were observed by microarrays in three cell groups. Cur significantly reversed the IR-induced IncRNA and mRNA expression signatures, shown by clustering analysis. Moreover, 116 of these IR-induced and Cur-reversed differentially expressed lncRNAs were obtained. Six lncRNAs (AF086415, AK095147, RP1-179N16.3, MUDENG, AK056098 and AK294004) were confirmed by qPCR. Furthermore, functional studies showed that 1ncRNA AK294004 exhibited a negative effect on cyclin D1 (CCND1), indicating that CCND1 might be a direct target of AK294004. IR-induced differentially expressed lncRNAs were reversed during Cur-enhanced radiosensitization in NPC cells, suggesting that lncRNAs have important functions in IR-induced radioresistance. Thus, Cur could serve as a good radiosensitizer.
\end{abstract}

\section{Introduction}

In Southeast China, nasopharyngeal carcinoma (NPC) is one of the most common malignancies of the head and neck that

Correspondence to: Dr Qin Fan or Professor Li Tong, College of Traditional Chinese Medicine, Southern Medical University, 1838 GuangZhou Avenue North, Guangzhou 510515, P.R. China E-mail: fqin@163.com

E-mail: zyxy2@fimmu.com

${ }^{*}$ Contributed equally

Key words: long non-coding RNA, curcumin, radiosensitivity, nasopharyngeal carcinoma, microarray can be effectively treated by radiotherapy $(1,2)$. However, a high proportion of patients with NPC exhibit radioresistance, which is the main risk factor that contributes to poor prognosis (3). Studies have revealed that increased radioresistance may be associated with various factors that participate in tumor development (4). Thus, the molecular mechanisms of radioresistance should be understood to provide opportunities for enhancing radiosensitivity and to develop a more effective anticancer strategy of NPC radiotherapy (5).

Curcumin (diferuloylmethane; Cur), a polyphenol from Curcuma longa rhizomes, is the major constituent of the yellow spice turmeric, a flavoring agent commonly used in Asian cooking (6). Cur also inhibits proliferation and angiogenesis in tumor cells to induce apoptosis or cell cycle arrest and cause tumor regression in pre-clinical models (7-9). In NPC, Cur has potent antitumor activity and radiosensitivity $(10,11)$; however, the exact molecular mechanism remains unclear.

Long non-coding RNAs (lncRNAs) are non-proteincoding transcripts that are longer than 200 nucleotides (12). They are pervasively transcribed with spatially and temporally regulated expression patterns (13). IncRNAs have important functions in gene expression regulation, dosage compensation, genomic imprinting, nuclear organization and compartmentalization, and nuclear-cytoplasmic trafficking (14-18). lncRNAs have continuously emerged as new contributing factors in cancer because they are involved in diverse biological processes and aberrantly expressed in various human cancers (17). IncRNAs also have potential functions in oncogenic (18) and tumor-suppressive pathways (19). IncRNAs also regulate gene expression at transcriptional, post-transcriptional, and epigenetic levels (20-22). Altered lncRNA expression may potentially enhance oncogenesis by altering some of these functions $(14,23)$. The differential lncRNA expressions can also indicate disease progression and function as predictors of patient outcomes.

In the present study, we demonstrated that Cur enhanced the radiosensitivity in NPC cell line CNE2 at an appropriate MTT concentration or with a clonogenic survival test. To determine the mechanism of radiosensitization, we performed a chip assay for CNE2 treated with irradiation (IR) and/or Cur. Numerous differentially expressed lncRNAs were identified, 
in which six lncRNAs were verified by qPCR. We observed that this response altered by IR was reversed by Cur in NPC cells. Our findings provide novel information on lncRNA expression profiles, in which Cur protected the cells from radiation toxicity, suggesting that this natural product may be an effective radiosensitizer or radioenhancer for managing patients with NPC.

\section{Materials and methods}

Cell culture. The present study was performed in human NPC cell lines [CNE-2; obtained from Sun Yat-sen University and had been described before (24)]. CNE-2 was maintained in Roswell Park Memorial Institute 1640 medium (RPMI-1640) supplemented with $10 \%$ fetal bovine serum (FBS; Invitrogen, USA) at $37^{\circ} \mathrm{C}$ in $5 \%$ carbon dioxide. Cur (Sigma-Aldrich, USA) was dissolved in $0.5 \%$ dimethyl sulphoxide (Sigma-Aldrich) and diluted with RPMI-1640 medium to the desired concentrations before use. The cells were divided into three groups [control group (CN); IR group (CX); and IR + Cur group (JX)] and irradiated linearly with X-rays at $6 \mathrm{MV}$ to deliver the indicated doses (2 Gy) at room temperature. The compensators used were $1.5 \mathrm{~cm}$ bolus. For the microarray, the sample was pooled in each group and the experiment was performed in triplicate.

Isolation of RNA. Total RNA was extracted using the TRIzol reagent (Invitrogen) according to the manufacturer's instructions. The RNA integrity number was checked to inspect RNA integration by an Agilent Bioanalyzer 2100 (Agilent Technologies, USA). Qualified total RNA was further purified using RNeasy mini kit (Qiagen, Germany) and RNase-free DNase set (Qiagen).

Preparation of array hybridization. The SBC 8x60K human lncRNA microarrays were custom designed using the Agilent eArray program according to the manufacturer's recommendations (https://earray.chem.agilent.com/earray). The microarray contained 31,171 mRNA probes, which were derived from the probe sequence for mRNA in Agilent 8x60K Whole Human Genome Oligo Microarray, and 29,971 lncRNA probes, which were designed by using an eArray-based system. The lncRNA sequence was derived from six databases, including LNCRNA-DB, NCBI_refseq, Ensembl, UCSC, NCBI_ unigene, and ncRNASCAN. After purification of labeled cRNAs, each slide was hybridized and washed according to the manufacturer's instructions (Agilent Technologies).

Data anaysis. Raw data were normalized by quantile algorithm on the Gene Spring 11.0 software (Agilent Technologies). lncRNAs and mRNAs with 'Present' or 'Marginal' (All Targets Value) flags in all of the groups were further subjected to data analysis. Differentially expressed lncRNAs and mRNAs were identified by fold change. Clustering was analyzed using the multi-experimental viewer (MeV) 4.6 and functional enrichment analysis was performed using DAVID's Functional Annotation Tool (http://david.abcc.ncifcrf.gov) (25).

Confirmation test of real-time quantitative RT-PCR. Total RNAs from tissues were extracted using TRIzol (Invitrogen) according to the manufacturer's instructions. Qualified total RNA was further purified by RNase-free DNase set (Qiagen). Reverse transcription was performed using a gene-specific primer and quantification was performed using the Quantitect SYBR Green PCR kit (Stratagene, USA) with an MX3005P multiplex quantitative PCR (qPCR) system (Stratagene) according to the manufacturer's instructions. GAPDH, the human housekeeping gene, was used for normalization. The relative lncRNA expression levels were calculated using the comparative $\Delta \Delta \mathrm{Ct}$ method as previously described (26). The fold changes were calculated according to $2^{-\Delta \Delta C t}$ equation. All of the primers used are listed in Table I.

RNA interference. In all, 20-30\% confluent CNE2 cells were transfected with $50 \mathrm{nM}$ of siRNAs using Lipofectamine 2000 (Invitrogen) following the manufacturer's direction. Two individual small interfering RNA (siRNAs) and scrambled negative control siRNA (siRNA-NC) were obtained from Invitrogen. The target sequences of AK294004 are the following: siRNA-1, 5-CUCCCUUCAACACUUCCUAAAUA-3 and siRNA-2, 5-AGCAGCAAACAAUGUGAAAGAGA-3. Thirty-six hours after transfection, cells were harvested for qRT-PCR (27).

Luciferase reporter assay. A 1,334-bp (1,794-3,127) fragment of CCND1 3'UTR containing whole complementry sequences of AK294004 two exons was amplified using the primer pairs $\mathrm{A}$, the sequence $(1,794-2,486 \mathrm{bp})$ of CCND1 3'UTR containing the complementry sequence of AK294004 exon 2 was amplified using the primer pairs $\mathrm{B}$, and the sequence (2,876-3,127 bp) of CCND1 3'UTR containing the complementry sequence of AK294004 exon 1 was amplified using the primer pairs $\mathrm{C}$ (Table I). Each fragment was respectively cloned downstream of the Renilla luciferase gene at the $\mathrm{XbaI}$ site in the pGL-3 promoter plasmid (Promega, USA). The entire 497-bp fragment of AK294004 was amplified using the the primer pairs D and was cloned at the KpnI and XhoI sites in the pcDNA3.1 $1^{+}$plasmid (Promega). The pGL3 constructs were designated as pGL3-W (whole sequence), pGL3-E1 (completed to exon 1) and pGL3-E2 (completed to exon 2) and the pcDNA3.1 construct was designated as pcDNA3-AK.

To facilitate cloning into each expression plasmid, the primers were designed to incorporate $X b a \mathrm{I}, K p n \mathrm{I}$ and $X h o \mathrm{I}$ sites at the 5 ' end (underlined in the primers above). HEK293 cells were co-transfected with 30 pmol of either pcDNA3-AK or pcDNA3-NC (empty vector control) and each pGL-construct using Lipofectamine 2000 (Invitrogen). Transfection efficiency was normalized by co-transfection with a firefly luciferase expressing plasmid. Luciferase activity was measured using the Promega dual-luciferase assay kit, in accordance with the instructions of the manufacturer. Relative protein levels were expressed as Renilla/firefly luciferase ratios. Each transfection was repeated twice in triplicates (27).

\section{Results}

LncRNA and mRNA microarray data. Array hybridization was performed using the SBC 8x60K human lncRNA microarrays. After quantile normalization of the raw data, the expression profiles of 29,971 lncRNAs and 31,171 mRNAs were obtained from the cells in the three groups. We identified differentially 
Table I. Primers for QPCR or cloning.

\begin{tabular}{|c|c|c|}
\hline Gene & Primer $\left(5^{\prime}-3^{\prime}\right)$ & Product (bp \\
\hline GAPDH & $\begin{array}{l}\text { Forward: ATCATCAGCAATGCCTCCTG } \\
\text { Reverse: ATGGACTGTGGTCATGAGTC }\end{array}$ & 102 \\
\hline AF086415 & $\begin{array}{l}\text { Forward: AGCGCGACTTCTCTGTCTCT } \\
\text { Reverse: GCAGAGGAGGAGACGCTGA }\end{array}$ & 115 \\
\hline AK095147 & $\begin{array}{l}\text { Forward: ACGAGTGACCGAAGCTGAAC } \\
\text { Reverse: GCACCATCCAGAGGGATTTA }\end{array}$ & 117 \\
\hline RP1-179N16.3 & $\begin{array}{l}\text { Forward: CGCGTTAGGAGATTCTGGAG } \\
\text { Reverse: AGGGTGGATACAGGCTCCTT }\end{array}$ & 105 \\
\hline AK056098 & $\begin{array}{l}\text { Forward: GGCCTCGGGGTAGAACTTAC } \\
\text { Reverse: CAAGCCTCCTGGTCTTTCTG }\end{array}$ & 143 \\
\hline AK294004 & $\begin{array}{l}\text { Forward: GTGCAACCAGAAATGCACAG } \\
\text { Reverse: ACGCTTTGTCTGTCGTGATG }\end{array}$ & 168 \\
\hline MUDENG & $\begin{array}{l}\text { Forward: ACTTTGTGGCACCGTGAGAT } \\
\text { Reverse: GGCCCACTAAATGCAGAGTC }\end{array}$ & 194 \\
\hline CCND1 & $\begin{array}{l}\text { Forward: GATCAAGTGTGACCCGGACT } \\
\text { Reverse: TCСТCСТCСТСТTCСТССТC }\end{array}$ & 129 \\
\hline Primer A & $\begin{array}{l}\text { Forward: gattatagaTGTAATTCTTGTAATTTTT } \\
\text { Reverse: gattatagaGCAGCAAACAATGTGAAAGA }\end{array}$ & \\
\hline Primer B & $\begin{array}{l}\text { Forward: gattatagaTCAACCATCCTGGCTGCGGCGT } \\
\text { Reverse: gattatagaTGCCGGTTACATGTTGGTGCT }\end{array}$ & \\
\hline Primer C & $\begin{array}{l}\text { Forward: gattatagaTGTAATTCTTGTAATTTTT } \\
\text { Reverse: gattatagaTGCCGGTTACATGTTGGTGCT }\end{array}$ & \\
\hline Primer D & $\begin{array}{l}\text { Forward: gatggtaccGCCGGTTACATGTTGGTGCT } \\
\text { Reverse: gatctcgagTGTAATTCTTGTAATTTTT }\end{array}$ & \\
\hline
\end{tabular}

Table II. Summary of differently expressed genes.

\begin{tabular}{lrrrrrr}
\hline & \multicolumn{2}{c}{ CX vs. CN } & \multicolumn{2}{c}{ JX vs. CN } & \multicolumn{2}{c}{ JX vs. CX } \\
\hline Up & 865 & $(592)$ & 623 & $(445)$ & 734 & $(615)$ \\
Down & 777 & $(712)$ & 579 & $(588)$ & 859 & $(832)$ \\
Total & 1,642 & $(1,304)$ & 1,202 & $(1,033)$ & 1,593 & $(1,447)$ \\
\hline
\end{tabular}

$\mathrm{CN}$, normal control; CX, ionizing radiation (IR); JX, IR + curcumin. LncRNA gene number (mRNA gene number).

expressed genes among the matched groups with a fold change $>2$. Table II summarizes the differentially expressed genes in each group.

Altered and reversed IncRNAs and mRNA expression. After clustering analysis was performed, JX and $\mathrm{CN}$ revealed seemingly similar expression signatures. The lncRNAs were altered by IR and Cur reversed this response and a similar trend was observed in the mRNA expression profile. Fig. 1 shows the heat maps of the expression ratios of IncRNAs and mRNAs among the JX, CX and CN groups. In addition, we focused on those altered expression genes induced by irradition while reversed by curcumin by a stronger raw signal screening. We obtained 116 lncRNAs, in which 76 were upregulated and 40 were downregulated in the CX group compared with those in the CN group. IncRNAs in the JX group were completely or partially reversed. We used the same screening method and obtained 178 differentially expressed mRNAs, in which 59 mRNAs were upregulated and 119 mRNAs were downregulated. Functional enrichment analysis of these differentially expressed genes was performed and clustered by DAVID's Functional Annotation Chart (25). Fig. 2 shows the functional annotation terms of the analysis.

Confirmation of some differentially expressed lncRNAs. We performed qPCR assays to confirm the expression patterns of some differentially expressed lncRNAs. qPCR results were consistent with the microarray analysis results of six lncRNAs (AF086415, AK095147, RP1-179N16.3, MUDENG, AK056098 and AK294004) in terms of regulation direction and significance. In particular, 0.29-fold downregulation in CX and 


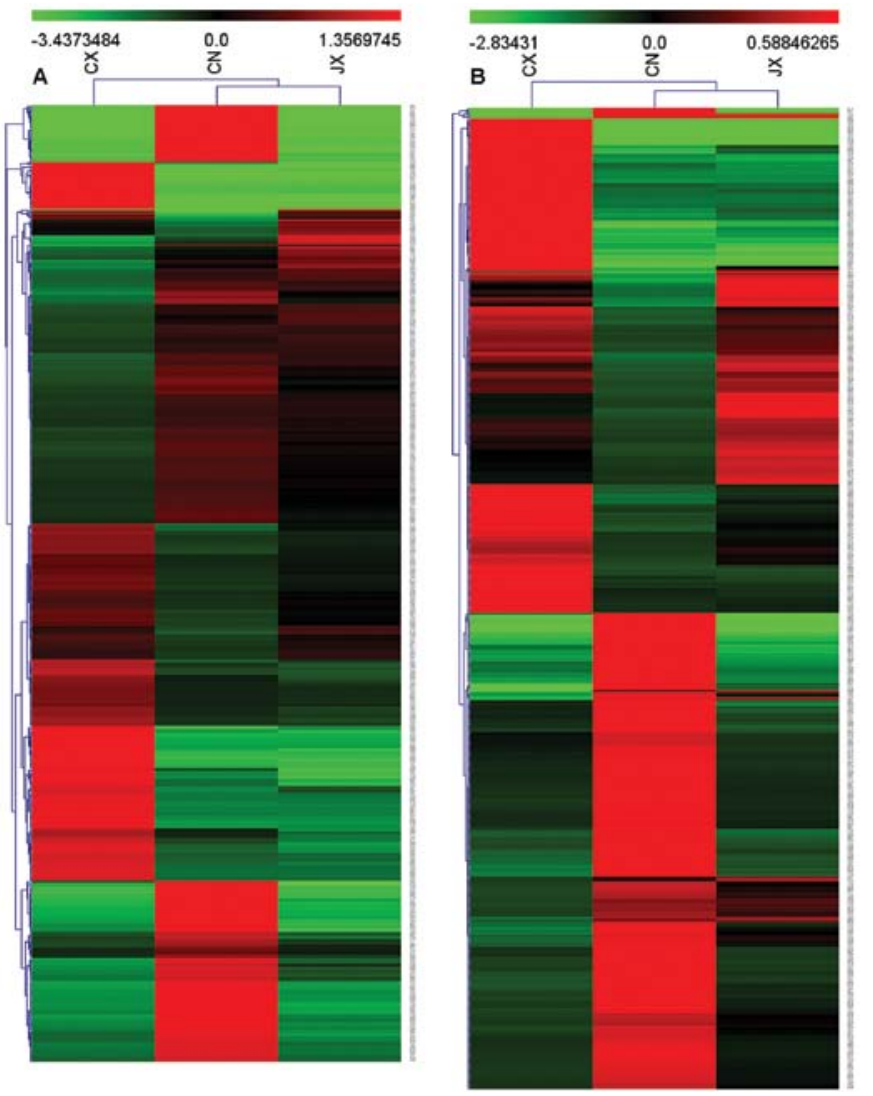

Figure 1. Heat maps of the expression ratios ( $\log 2$ scale) of $\operatorname{lncRNAs}(\mathrm{A})$ and mRNAs (B) in different groups of CNE2 cells. Red and green indicate high and low relative expressions, respectively. $\mathrm{CN}$, control group; CX, IR group; and JX, IR + Cur group.

0.78-fold reversal in JX were observed in AF086415 (0.38- and 0.59-fold in microarray analysis, respectively). For AK095147, 0.31 -fold downregulation in CX and 0.92-fold of reversal in JX were observed (0.44- and 1.04-fold in microarray analysis, respectively). For RP1-179N16.3, 5.25-fold upregulation in $\mathrm{CX}$ and 1.54-fold reversal in JX were observed (6.96- and 1.04-fold in microarray analysis, respectively). For MUDENG, 4.01-fold upregulation in CX and 1.34-fold reversal in JX were observed (4.98- and 0.85-fold in microarray analysis, respectively). For AK056098, 3.81-fold upregulation in CX and 1.08-fold reversal in JX were observed (3.14- and 1.16-fold in microarray analysis, respectively). For AK294004, 2.88-fold upregulation in CX and 1.21-fold reversal in JX were observed (2.86- and 0.91-fold in microarray analysis, respectively. All of the fold changes were compared with $\mathrm{CN}$ (Fig. 3).

Effect of interaction between AK294004 and 3'UTR of CCND1. AK294004 exhibited a negative effect on CCND1. For AK294004, 2.88-fold upregulation in CX and 1.21-fold reversal in JX were observed by qPCR, and for CCND1, 0.26-fold downregulation in CX and 0.61-fold reversal in JX were observed (compared with CN, Fig. 4).

To investigate the functional effects of AK294004 in NPC cells, we modulated its expression through RNA interference and overexpression experiments. pCDNA3-AK and two individual AK294004 siRNAs were transfected into CNE2 cells. qPCR analysis of AK204004 and CCND1 levels was performed
A

cluster

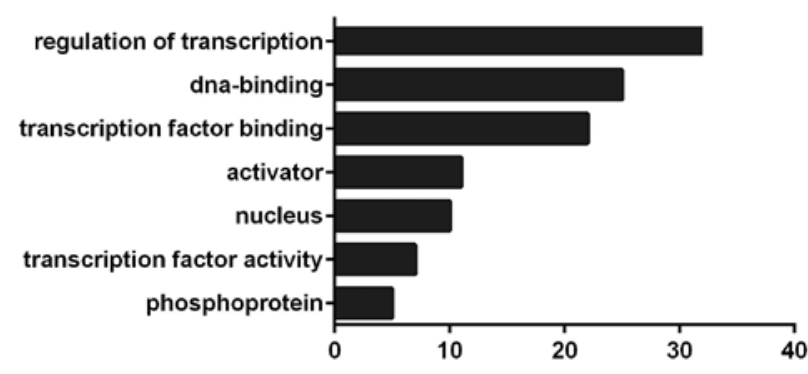

B

cluster

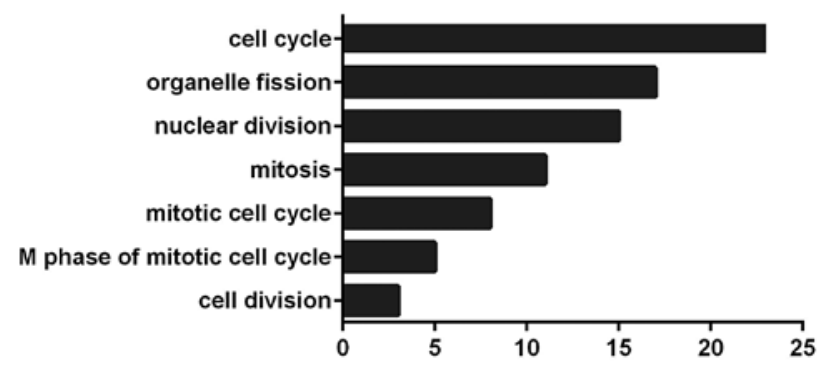

Figure 2. Functional enrichment analysis on IR-induced and Cur-reversed differentially regulated genes. Annotation of $116 \operatorname{lncRNAs}$ (A) and 178 mRNAs (B) was performed by utilizing the DAVID Functional Annotation Chart (25).

A

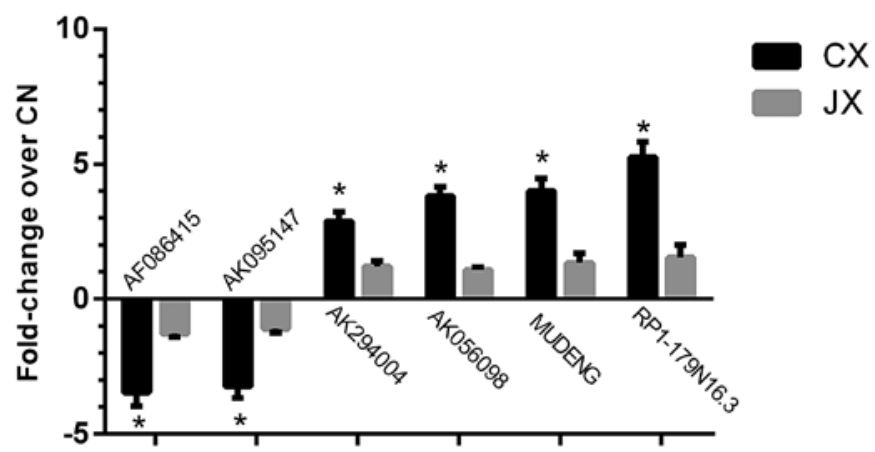

B

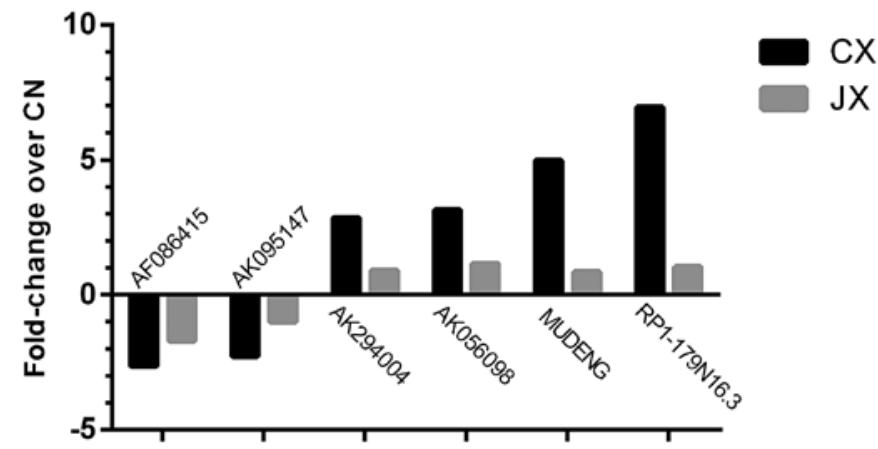

Figure 3. Comparison between microarray data and qPCR results. AF086415, AK095147, RP1-179N16.3, MUDENG, AK056098 and AK294004 differentially expressed in CX and reversed in JX by microarray (B) were validated by qPCR (A). The heights of the columns in the chart represent the fold changes. The columns below the $\mathrm{x}$-axis represent downregulation. qPCR results show mean $\pm \mathrm{SD}\left(\mathrm{n}=3\right.$; t-test; ${ }^{*} \mathrm{P}<0.05$, compared with $\left.\mathrm{CN}\right)$. 


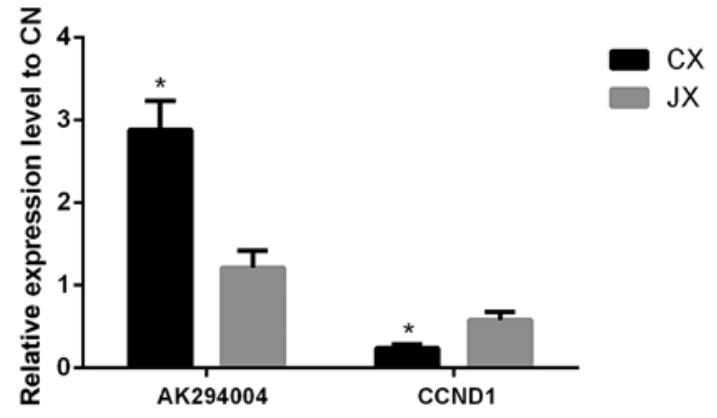

Figure 4. Negative relationship between AK294004 and CCND1 expression by qPCR. The heights of the columns in the chart represent the relative fold changes. Results show mean $\pm \mathrm{SD}\left(\mathrm{n}=3\right.$; t-test; ${ }^{*} \mathrm{P}<0.05$, compared with $\left.\mathrm{CN}\right)$. No absolute relationship exists between lncRNA and mRNA columns.

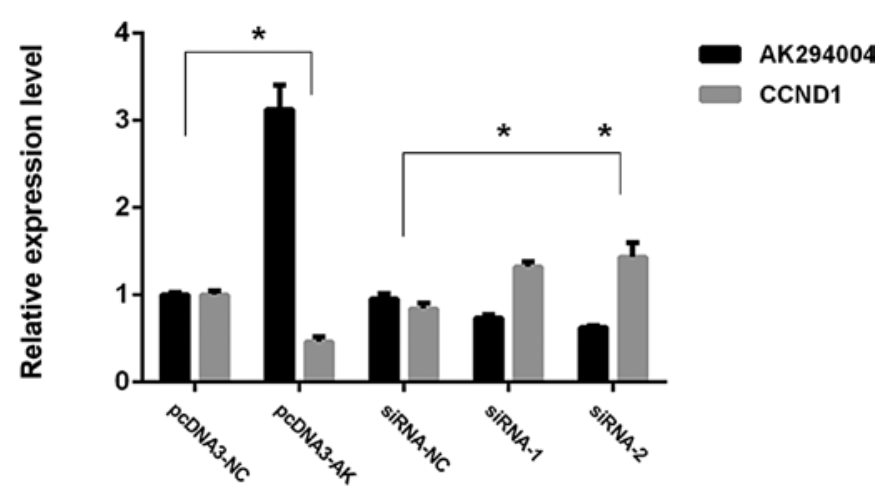

Figure 5. Modulated expression levels of AK294004 and CCND1 by qPCR. pCDNA3-AK and two individual AK294004 siRNAs were transfected into CNE2 cells. qPCR analysis of AK204004 and CCND1 levels was performed $36 \mathrm{~h}$ post-transfection. Data represent mean \pm SD of the three independent experiments and each experiment was performed in triplicate. " $\mathrm{P}<0.05$.
36 h post-transfection. As shown in Fig. 5, for overexpression experiments, AK204004 expression was increased 3.16-fold while CCND1 expression was decreased by $46 \%$ in pCDNA3AK cells, compared with control cells (pCDNA3-NC). For RNA interference experiments, when compared with control cells (siRNA-NC), AK294004 expression was knocked down $67 \%$ by siRNA-2, and $75 \%$ by siRNA-1, while CCND1 expression was increased 1.34- and 1.48-fold, respectively.

A luciferase-based reporter was constructed to evaluate the effect of AK294004 direct binding to the putative target sites on the 3'UTR of CCND1. To substantiate the assumption that AK294004 can directly repress CCND1, the reporter construct pGL3-vector or pGL3-W, pGL3-E1 and pGL3-E2 was co-transfected with pcDNA3-AK and pcDNA3-NC to HEK 293 cells. Luciferase activity was then assayed. As shown in Fig. 6, for pGL3-W or pGL3-E1 construct, pcDNA3-AK significantly lowered luciferase activity compared with pcDNA3-NC. There was no different luciferase activity observed between the pGL3-vector and pGL3-E2 constructs.

These findings support the hypothesis that lncRNA AK294004 directly targets CCND1 expression by its exon 1 part, but not the exon 2 part, thus leading to the decreased CCND1 expression through some inhibition mechanism.

\section{Discussion}

Radiotherapy is considered one of the most effective treatments for patients with NPC, and radioresistance is the main risk factor that contributes to poor prognosis (2). Radioresistance occurs in primary IR treatment and the survived cells may be more resistant to the second IR treatment, thereby leading to the failure of radiotherapy $(2,28,29)$. In this regard, the

A
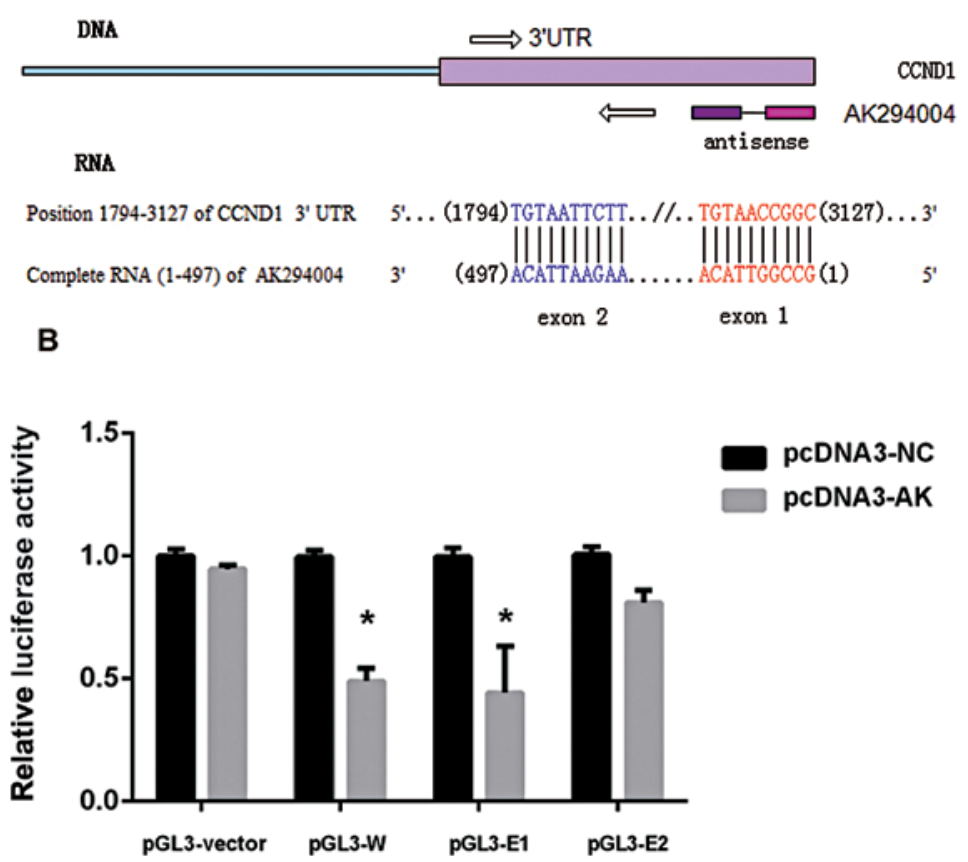

Figure 6. Effect of the putative 1ncRNA binding site derived from the CCND1 3'UTR on luciferase expression. (A) Schematic of the potential AK294004 binding site containing CCND1 3'UTR. (B) Luciferase activity in HEK293 cells transfected with pcDNA3-NC and pcDNA3-AK along with pGL3-vector or pGL3-W, pGL3-E2 and pGL3-E2 36 h post-transfection. Luciferase activity was measured using the Promega dual-luciferase assay kit, according to the instructions of the manufacturer. Data represent mean $\pm \mathrm{SD}$ of the three independent experiments and each experiment was performed in triplicate. ${ }^{*} \mathrm{P}<0.05$. 
exact molecules and signaling pathway involved in radiosensitization should be determined to develop target therapy and enhance the efficacy of radiation. In this study, we observed that IR-induced differentially expressed lncRNAs were almost reversed by Cur. This result is consistent with our hypothesis, in which Cur enhances radiosensitivity through the reversal of effective molecules (7,30,31). For example, AK294004, a natural antisense IncRNA, exhibited 2.86-fold upregulation in $\mathrm{CX}$ (compare with $\mathrm{CN}$ ), whereas a reversal at 0.32 -fold downregulation by Cur was observed in JX (compare with $\mathrm{CX}$ ). This finding was further confirmed by qPCR. IncRNA may have an important function in IR-induced radioresistance.

Cur regulates the gene expression involved in survival, proliferation, angiogenesis, invasion and metastasis. This phytochemical also modulates various mechanisms that are associated with radioresistance, including the following: downregulating COX-2, MRP, Bcl-2, and survivin expression; inhibiting PI3K/AKT activation; suppressing growth factor signaling pathways; and inhibiting STAT3 activation (32-34). In this study, we demonstrated that Cur enhanced radiosensitivity in the NPC cell line CNE2 at $10 \mu \mathrm{mol} / 1$ by MTT or clonogenic survival test (35) before we performed the array test (data not shown), although Cur exhibited higher anti-proliferative effects when used alone at a concentration of 20 or $40 \mu \mathrm{mol} / \mathrm{l}$. Considering the cytotoxicity of Cur and IR, a concentration of $10 \mu \mathrm{mol} / 1$ was more suitable as a radioenhancer. Therefore, no significant data were obtained by conjoint analysis with other groups at particular time-points, although the array of JN (Cur group) was performed (data not shown). Further analysis need to be performed to reveal other chemical mechanisms for Cur. Furthermore, the optimal IR dosage of 2 Gy and the Cur pretreatment time of $6 \mathrm{~h}$ were confirmed for the succeeding study (data not shown).

The mammalian genome clearly encodes numerous lncRNAs that are highly conserved and biologically functional (36). Expression patterns have suggested that these lncRNAs are involved in diverse biological processes, including cell cycle regulation, innate immunity, and pluripotency (37), but current understanding on the functions of lncRNAs is limited. In this study, 116 differentially expressed lncRNAs were expressed site-specifically, such as intergenic, intronic antisense, natural antisense, bidirectional, and intron sense overlapping. We used the DAVID Functional Annotation Chart (25) for the functional enrichment analysis of these differentially expressed genes. In this study, the most significant functional annotation terms of 116 lncRNAs were transcription regulation, DNA binding, transcription factor binding, activator and nucleus (Fig. 2A). For 178 mRNAs, the functional annotation terms were cell cycle, organelle fission, nuclear division, mitosis, mitotic cell cycle and $\mathrm{M}$ phase of the mitotic cell cycle (Fig. 2B). No direct relationship was found between the altered lncRNA and mRNA expressions, indicating that lncRNA performed a biological function via a complex regulatory mechanism instead of directly targeting mRNA during the Cur-induced radiosensitization involved in NPC.

AK294004, a natural antisense lncRNA that completely complements the terminal end of the 3 ' untranslated region of CCND1 mRNA, exhibited a negative effect on CCND1, an important molecule of the cell cycle and DNA repair. CCND1 is downregulated during IR-induced DNA damage $(17,38)$. In this study, we observed the IR-induced altered regulation and the Cur-induced reversal of either AK294004 or CCND1 that were consequently confirmed by qPCR (Fig. 4). Moreover, luciferase reporter assay and modulated expression experiments indicated that CCND1 might be a direct target of AK294004, however, it needed to be further determined how these lcnRNAs and mRNAs interact with one another.

In general, the cells respond to IR-induced biological process, such as DNA damage repair, cell cycle arrest, and so on $(33,39-41)$. In this study, we performed the microarray assay at $3 \mathrm{~h}$ post-IR. We also performed qPCR to validate the altered lncRNA expression at different time-points until $48 \mathrm{~h}$ post-IR was reached in parallel cell groups (data not shown). The microarray results were consistent with the qPCR data, particularly at 3-12 h checkpoint but slightly differed after $24 \mathrm{~h}$. These differences in responses may be attributed to different mechanisms of IncRNA performance.

In conclusion, we demonstrated the mechanism by which Cur enhanced radiosensitivity in NPC cells that involved differentially expressed lncRNAs and provided better understanding of chemically-mediated radiosensitization. The function of Cur-induced lncRNA reversal should be fully understood to provide a new and more effective radiotherapeutic treatment for patients with NPC by using natural products.

\section{Acknowledgements}

This study was supported by National Natural Science Foundation of China (grant no. 81173616) and New Technology Project of Nanfang Hospital (grant no. 201103). We thank medical personnel Jiabin Liu and Huarui Niu of NanFang Hospital for providing X-ray radiation equipment.

\section{References}

1. Ou J, Pan F, Geng P, et al: Silencing fibronectin extra domain A enhances radiosensitivity in nasopharyngeal carcinomas involving an FAK/Akt/JNK pathway. Int J Radiat Oncol Biol Phys 82: e685-e691, 2012.

2. Lu ZX, Ma XQ, Yang LF, et al: DNAzymes targeted to EBV-encoded latent membrane protein-1 induce apoptosis and enhance radiosensitivity in nasopharyngeal carcinoma. Cancer Lett 265: 226-238, 2008.

3. Ou J, Luan W, Deng J, Sa R and Liang H: $\alpha$ v integrin induces multicellular radioresistance in human nasopharyngeal carcinoma via activating SAPK/JNK pathway. PLoS One 7: e38737, 2012.

4. Bernier J: A multidisciplinary approach to squamous cell carcinomas of the head and neck: an update. Curr Opin Oncol 20: 249-255, 2008.

5. Feng XP, Yi H, Li MY, et al: Identification of biomarkers for predicting nasopharyngeal carcinoma response to radiotherapy by proteomics. Cancer Res 70: 3450-3462, 2010.

6. Sandur SK, Deorukhkar A, Pandey MK, et al: Curcumin modulates the radiosensitivity of colorectal cancer cells by suppressing constitutive and inducible NF-kappaB activity. Int J Radiat Oncol Biol Phys 75: 534-542, 2009.

7. Shishodia S, Amin HM, Lai R and Aggarwal BB: Curcumin (diferuloylmethane) inhibits constitutive NF-kappaB activation, induces G1/S arrest, suppresses proliferation, and induces apoptosis in mantle cell lymphoma. Biochem Pharmacol 70: 700-713, 2005.

8. Li L, Aggarwal BB, Shishodia S, Abbruzzese J and Kurzrock R: Nuclear factor-kappaB and IkappaB kinase are constitutively active in human pancreatic cells, and their down-regulation by curcumin (diferuloylmethane) is associated with the suppression of proliferation and the induction of apoptosis. Cancer 101: 2351-2362, 2004 
9. Bharti AC, Shishodia S, Reuben JM, et al: Nuclear factorkappaB and STAT3 are constitutively active in CD138 $8^{+}$cells derived from multiple myeloma patients, and suppression of these transcription factors leads to apoptosis. Blood 103: 3175 3184,2004

10. Wang X, Xia X, Xu C, et al: Ultrasound-induced cell death of nasopharyngeal carcinoma cells in the presence of curcumin Integr Cancer Ther 10: 70-76, 2011.

11. Wong TS, Chan WS, Li CH, et al: Curcumin alters the migratory phenotype of nasopharyngeal carcinoma cells through up-regulation of E-cadherin. Anticancer Res 30: 2851-2856, 2010.

12. Zhang X, Sun S, Pu JK, et al: Long non-coding RNA expression profiles predict clinical phenotypes in glioma. Neurobiol Dis 48 : $1-8,2012$.

13. Kaikkonen MU, Lam MT and Glass CK: Non-coding RNAs as regulators of gene expression and epigenetics. Cardiovasc Res 90: 430-440, 2011

14. Mercer TR, Dinger ME and Mattick JS: Long non-coding RNAs: insights into functions. Nat Rev Genet 10: 155-159, 2009

15. Wang KC and Chang HY: Molecular mechanisms of long noncoding RNAs. Mol Cell 43: 904-914, 2011.

16. Nagano $T$ and Fraser P: No-nonsense functions for long noncoding RNAs. Cell 145: 178-181, 2011.

17. Ozgur E, Mert U, Isin M, Okutan M, Dalay N and Gezer U: Differential expression of long non-coding RNAs during genotoxic stress-induced apoptosis in HeLa and MCF-7 cells. Clin Exp Med 13: 119-126, 2013.

18. Barsyte-Lovejoy D, Lau SK, Boutros PC, et al: The c-Myc oncogene directly induces the H19 noncoding RNA by allelespecific binding to potentiate tumorigenesis. Cancer Res 66 : 5330-5337, 2006

19. Zhou Y, Zhong Y, Wang Y, et al: Activation of p53 by MEG3 non-coding RNA. J Biol Chem 282: 24731-24742, 2007.

20. Mariner PD, Walters RD, Espinoza CA, et al: Human Alu RNA is a modular transacting repressor of mRNA transcription during heat shock. Mol Cell 29: 499-509, 2008.

21. Beltran M, Puig I, Pena C, et al: A natural antisense transcript regulates Zeb2/Sip1 gene expression during Snail1-induced epithelial-mesenchymal transition. Genes Dev 22: 756-769, 2008.

22. Gupta RA, Shah N, Wang KC, et al: Long non-coding RNA HOTAIR reprograms chromatin state to promote cancer metastasis. Nature 464: 1071-1076, 2010.

23. Huarte $M$ and Rinn JL: Large non-coding RNAs: missing links in cancer? Hum Mol Genet 19: R152-R161, 2010.

24. Wang J, Guo LP, Chen LZ, Zeng YX and Lu SH: Identification of cancer stem cell-like side population cells in human nasopharyngeal carcinoma cell line. Cancer Res 67: 3716-3724, 2007.

25. Huang da W, Sherman BT and Lempicki RA: Bioinformatics enrichment tools: paths toward the comprehensive functional analysis of large gene lists. Nucleic Acids Res 37: 1-13, 2009

26. Fan Q, He M, Deng X, et al: Derepression of c-Fos caused by MicroRNA-139 down-regulation contributes to the metastasis of human hepatocellular carcinoma. Cell Biochem Funct 31: 319-324, 2013
27. Liu Y, Cai H, Liu J, et al: A miR-151 binding site polymorphism in the 3'-untranslated region of the cyclin E1 gene associated with nasopharyngeal carcinoma. Biochem Biophys Res Commun 432: 660-665, 2013

28. Pearce AG, Segura TM, Rintala AC, Rintala-Maki ND and Lee $\mathrm{H}$ : The generation and characterization of a radiationresistant model system to study radioresistance in human breast cancer cells. Radiat Res 156: 739-750, 2001.

29. Baldwin AS: Control of oncogenesis and cancer therapy resistance by the transcription factor NF-kappaB. J Clin Invest 107 241-246, 2001

30. Singh S and Aggarwal BB: Activation of transcription factor NF-kappa B is suppressed by curcumin (diferuloylmethane) [corrected]. J Biol Chem 270: 24995-25000, 1995.

31. Li JY, Li YY, Jin W, Yang Q, Shao ZM and Tian XS: ABT-737 reverses the acquired radioresistance of breast cancer cells by targeting Bcl-2 and Bcl-xL. J Exp Clin Cancer Res 31: 102, 2012.

32. Kunnumakkara AB, Diagaradjane P, Guha S, et al: Curcumin sensitizes human colorectal cancer xenografts in nude mice to gamma-radiation by targeting nuclear factor-kappaB-regulated gene products. Clin Cancer Res 14: 2128-2136, 2008.

33. Javvadi P, Segan AT, Tuttle SW and Koumenis C: The chemopreventive agent curcumin is a potent radiosensitizer of human cervical tumor cells via increased reactive oxygen species production and overactivation of the mitogen-activated protein kinase pathway. Mol Pharmacol 73: 1491-1501, 2008.

34. Narang $\mathrm{H}$ and Krishna $\mathrm{M}$ : Inhibition of radiation induced nitration by curcumin and nicotinamide in mouse macrophages. Mol Cell Biochem 276: 7-13, 2005.

35. Hannoun-Levi JM, Chand-Fouche ME, Dejean C and Courdi A Dose gradient impact on equivalent dose at 2 Gy for high dose rate interstitial brachytherapy. J Contemp Brachytherapy 4: 14-20, 2012.

36. Khalil AM, Guttman M, Huarte M, et al: Many human large intergenic noncoding RNAs associate with chromatin-modifying complexes and affect gene expression. Proc Natl Acad Sci USA 106: 11667-11672, 2009.

37. Guttman M, Amit I, Garber M, et al: Chromatin signature reveals over a thousand highly conserved large non-coding RNAs in mammals. Nature 458: 223-227, 2009.

38. Wang X, Arai S, Song X, et al: Induced ncRNAs allosterically modify RNA-binding proteins in cis to inhibit transcription. Nature 454: 126-130, 2008

39. Aravindan N, Veeraraghavan J, Madhusoodhanan R, Herman TS and Natarajan M: Curcumin regulates low-linear energy transfer gamma-radiation-induced NFkappaB-dependent telomerase activity in human neuroblastoma cells. Int J Radiat Oncol Biol Phys 79: 1206-1215, 2011.

40. Forrester HB, Li J, Hovan D, Ivashkevich AN and Sprung CN: DNA repair genes: alternative transcription and gene expression at the exon level in response to the DNA damaging agent, ionizing radiation. PLoS One 7: e53358, 2012.

41. Lan ML, Acharya MM, Tran KK, et al: Characterizing the radioresponse of pluripotent and multipotent human stem cells. PLoS One 7: e50048, 2012. 